\title{
SINGULAR INTEGRALS RELATED TO HOMOGENEOUS MAPPING WITH ROUGH KERNELS ON PRODUCT SPACES
}

\author{
H. AL-QASSEM AND M. ALI
}

\begin{abstract}
In this paper, we study the $L^{p}$ mapping properties of singular integral operators related to homogeneous mappings on product spaces with kernels which belong to block spaces. Our results extend as well as improve some known results on singular integrals.
\end{abstract}

\section{Introduction}

Suppose that $\mathbf{S}^{d-1}(d=n$ or $m)$ is the unit sphere in $\mathbf{R}^{d}$ equipped with the normalized Lebesgue measure $d \sigma=d \sigma(\cdot)$ which normalized so that $\sigma\left(\mathbf{S}^{d-1}\right)=1$. For a nonzero point $x \in \mathbf{R}^{d}$, we let $x^{\prime}=x /|x|$. For $n, m \geq 2$, let $K_{\Omega}(\cdot, \cdot)$ be the singular kernel on $\mathbf{R}^{n} \times \mathbf{R}^{m}$ given by

$$
K_{\Omega}(u, v)=\Omega\left(u^{\prime}, v^{\prime}\right)|u|^{-n}|v|^{-m},
$$

where $\Omega \in L^{1}\left(\mathbf{S}^{n-1} \times \mathbf{S}^{m-1}\right)$ and satisfies the cancellation conditions

$$
\int_{\mathbf{S}^{n-1}} \Omega(u, \cdot) d \sigma(u)=\int_{\mathbf{S}^{m-1}} \Omega(\cdot, v) d \sigma(v)=0 .
$$

For suitable mappings $\Phi: \mathbf{R}^{n} \longrightarrow \mathbf{R}^{N}$ and $\Psi: \mathbf{R}^{m} \longrightarrow \mathbf{R}^{M}$, define the singular integral operator $T_{\Phi, \Psi, \Omega}$ and its related maximal truncated operator $T_{\Phi, \Psi, \Omega}^{*}$ on the product space $\mathbf{R}^{n} \times \mathbf{R}^{m}$ by

$$
\begin{aligned}
& T_{\Phi, \Psi, \Omega} f(x, y)=\text { p.v. } \int_{\mathbf{R}^{n} \times \mathbf{R}^{m}} f(x-\Phi(u), y-\Psi(v)) K_{\Omega}(u, v) d u d v, \\
& T_{\Phi, \Psi, \Omega}^{*} f(x, y)=\sup _{\varepsilon_{1}, \varepsilon_{2}>0}\left|\int_{\left\{|u| \geq \varepsilon_{1},|v| \geq \varepsilon_{2}\right\}} f(x-\Phi(u), y-\Psi(v)) K_{\Omega}(u, v) d u d v\right|
\end{aligned}
$$

for $f \in \mathscr{S}\left(\mathbf{R}^{N} \times \mathbf{R}^{M}\right)$.

When $n=N, m=M, \Phi(x) \equiv x$ and $\Psi(y) \equiv y$ for $(x, y) \in \mathbf{R}^{n} \times \mathbf{R}^{m}$, the operators $T_{\Omega}=T_{\Phi, \Psi, \Omega}$ and $T_{\Omega}^{*}=T_{\Phi, \Psi, \Omega}^{*}$ become the classical Calderón-Zygmund singular integral operator and its

Received December 28, 2004.

2000 Mathematics Subject Classification. Primary 42B20; Secondary 42B15, 42B2..

Key words and phrases. Singular integrals, oscillatory integrals, Fourier transform, product spaces, rough kernels, block spaces. 
corresponding maximal truncated operator on the product space $\mathbf{R}^{n} \times \mathbf{R}^{m}$ given by

$$
\begin{aligned}
& T_{\Omega} f(x)=\text { p.v. } \int_{\mathbf{R}^{n} \times \mathbf{R}^{m}} f(x-u, y-v) K_{\Omega}(u, v) d u d v, \\
& T_{\Omega}^{*} f(x)=\sup _{\varepsilon_{1}, \varepsilon_{2}>0}\left|\int_{\left\{|u| \geq \varepsilon_{1},|v| \geq \varepsilon_{2}\right\}} f(x-u, y-v) K_{\Omega}(u, v) d u d v\right| .
\end{aligned}
$$

The study of the $L^{p}$ mapping properties of $T_{\Omega}$ and its extensions has attracted the attention of many authors. We refer the readers to [3], [5], [6], [8], [9], among others. Let us now recall some known results. R. Fefferman and E. Stein in [9] showed that if $\Omega$ satisfies certain Lipschitz conditions, then the operators $T_{\Omega}$ and $T_{\Omega}^{*}$ are bounded in $L^{p}\left(\mathbf{R}^{n} \times \mathbf{R}^{m}\right)$ for $p \in(1, \infty)$. In [5], Duoandikoetxea improved the results in [9] by showing that $T_{\Omega}$ is bounded on $L^{p}$ for $1<p<\infty$ if $\Omega \in L^{q}\left(\mathbf{S}^{n-1} \times \mathbf{S}^{m-1}\right)$, with $q>1$. In [6], Fan, Guo and Pan improved the result in [5] by showing that the $L^{p}(1<p<\infty)$ continues to hold even $\Omega$ belongs to the block space $B_{q}^{(0,1)}\left(\mathbf{S}^{n-1} \times \mathbf{S}^{m-1}\right)$ for some $q>1$ (for $p=2$, this result was proved first by Jiang and Lu in [10]). In [3], Al-Qassem and Pan proved the $L^{p}(1<p<\infty)$ boundedness of the more general class of operators $T_{\Phi, \Psi, \Omega}$ and $T_{\Phi, \Psi, \Omega}^{*}$ if $\Omega \in B_{q}^{(0,1)}\left(\mathbf{S}^{n-1} \times \mathbf{S}^{m-1}\right)$ for some $q>1$ and $\Phi, \Psi$ are polynomial mappings on $\mathbf{R}^{n}$ and $\mathbf{R}^{m}$, respectively.

Our main purpose in this paper is to investigate the $L^{p}$ boundedness of $T_{\Phi, \Psi, \Omega}$ and $T_{\Phi, \Psi, \Omega}^{*}$ if $\Omega \in B_{q}^{(0,1)}\left(\mathbf{S}^{n-1} \times \mathbf{S}^{m-1}\right)$ and $\Phi$ and $\Psi$ are homogeneous mappings. To state our main result, we need first the following definition.

For $d=\left(d_{1}, \ldots, d_{l}\right) \in \mathbf{R}^{l}$, define the family of dilations $\left\{\delta_{t}\right\}_{t>0}$ on $\mathbf{R}^{l}$ by

$$
\delta_{t}\left(x_{1}, \ldots, x_{l}\right)=\left(t^{d_{1}} x_{1}, \ldots, t^{d_{l}} x_{l}\right) .
$$

We say that a mapping $\Phi: \mathbf{R}^{n} \longrightarrow \mathbf{R}^{l}$ is homogeneous of degree $d$ if

$$
\Phi(t x)=\delta_{t}(\Phi(x))
$$

holds for all $x \in \mathbf{R}^{n} \backslash\{0\}$ and $t>0$.

Now, the following is our main result in this paper:

Theorem 1.1. Let $T_{\Phi, \Psi, \Omega}$ and $T_{\Phi, \Psi, \Omega}^{*}$ be given by (1.3) and (1.4), respectively. Suppose that $\Omega \in$ $B_{q}^{(0,1)}\left(\mathbf{S}^{n-1} \times \mathbf{S}^{m-1}\right)$ for some $q>1$ and satisfies (1.2). Let $\Phi: \mathbf{R}^{n} \longrightarrow \mathbf{R}^{N}$ and $\Psi: \mathbf{R}^{m} \longrightarrow \mathbf{R}^{M}$ be homogeneous mappings of degrees $d=\left(d_{1}, \ldots, d_{N}\right)$ and $h=\left(h_{1}, \ldots, h_{M}\right)$, respectively with $d_{l}$, $h_{r} \neq 0$ for $1 \leq l \leq N$ and $1 \leq r \leq M$. Assume that $\Phi \mid \mathbf{S}^{n-1}$ and $\Psi \mid \mathbf{S}^{m-1}$ are real-analytic. Then there exists a positive constant $C_{p}>0$ such that

$$
\left\|T_{\Phi, \Psi, \Omega}(f)\right\|_{p} \leq C_{p}\|f\|_{p},
$$

and

$$
\left\|T_{\Phi, \Psi, \Omega}^{*}(f)\right\|_{p} \leq C_{p}\|f\|_{p}
$$

for any $f \in L^{p}\left(\mathbf{R}^{n} \times \mathbf{R}^{m}\right)$ with $1<p<\infty$. 
Remarks. (1) We point out that on $\mathbf{S}^{n-1} \times \mathbf{S}^{m-1}$, for any $q>1$ and $v>-1$, the following inclusion holds and is proper:

$$
\bigcup_{r>1} L^{r}\left(\mathbf{S}^{n-1} \times \mathbf{S}^{m-1}\right) \subset B_{q}^{(0, v)}\left(\mathbf{S}^{n-1} \times \mathbf{S}^{m-1}\right) .
$$

The question with regard to the relationship between $B_{q}^{(0, \alpha-1)}\left(\mathbf{S}^{n-1} \times \mathbf{S}^{m-1}\right)$ and $L\left(\log ^{+} L\right)^{\alpha} L\left(\mathbf{S}^{n-1} \times\right.$ $\mathbf{S}^{m-1}$ ) (for $\alpha>0$ ) remains open.

(2) One observes that Theorem 1.1 represents an improvement over the corresponding results in [9] and an extension of the main result in [6].

(3) We remark that the one parameter case of Theorem 1.1 was studied by many authors (see for example, [7], [4], [2]). Also, we point that a similar result to Theorem 1.1 was obtained in [1] when $\Omega$ belongs to the class $\Omega \in L\left(\log ^{+} L\right)^{2}\left(\mathbf{S}^{n-1} \times \mathbf{S}^{m-1}\right)$.

The paper is organized as follows. A few lemmas will be recalled or proved in Section 2. The proof of Theorem 1.1 can be found in Section 3.

Throughout this paper, the letter $C$ will denote a bounded positive constant that may vary at each occurrence but independent of the essential variables.

\section{Definitions and lemmas}

The block spaces originated in the work of M. H. Taibleson and G. Weiss on the convergence of the Fourier series (see [15]) in connection with the developments of the real Hardy spaces. Below we shall recall the definition of block spaces on $\mathbf{S}^{n-1} \times \mathbf{S}^{m-1}$. For further background information about the theory of spaces generated by blocks and its applications to harmonic analysis one can consult the book [12].

The special class of block spaces $B_{q}^{(0, v)}\left(\mathbf{S}^{n-1} \times \mathbf{S}^{m-1}\right.$ ) (for $v>-1$ and $q>1$ ) was introduced by Jiang and Lu with respect to the study of singular integral operators on product domains [10].

Definition 2.1. A $q$-block on $\mathbf{S}^{n-1} \times \mathbf{S}^{m-1}$ is an $L^{q}(1<q \leq \infty)$ function $b(x, y)$ that satisfies

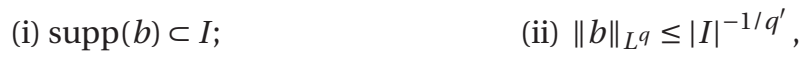

where $|\cdot|$ denotes the product measure on $\mathbf{S}^{n-1} \times \mathbf{S}^{m-1}$, and $I$ is an interval on $\mathbf{S}^{n-1} \times \mathbf{S}^{m-1}$, i.e.,

$$
I=\left\{x^{\prime} \in \mathbf{S}^{n-1}:\left|x^{\prime}-x_{0}^{\prime}\right|<\alpha\right\} \times\left\{y^{\prime} \in \mathbf{S}^{m-1}:\left|y^{\prime}-y_{0}^{\prime}\right|<\beta\right\}
$$

for some $\alpha, \beta>0$ and $\left(x_{0}^{\prime}, y_{0}^{\prime}\right) \in \mathbf{S}^{n-1} \times \mathbf{S}^{m-1}$.

Definition 2.2. The block space $B_{q}^{(0, v)}=B_{q}^{(0, v)}\left(\mathbf{S}^{n-1} \times \mathbf{S}^{m-1}\right)$ is defined by

$$
B_{q}^{(0, v)}=\left\{\Omega \in L^{1}\left(\mathbf{S}^{n-1} \times \mathbf{S}^{m-1}\right): \Omega=\sum_{\mu=1}^{\infty} C_{\mu} b_{\mu}, M_{q}^{(0, v)}\left(\left\{C_{\mu}\right\}\right)<\infty\right\},
$$

where each $C_{\mu}$ is a complex number; each $b_{\mu}$ is a $q$-block supported on a interval $I_{\mu}$ on $\mathbf{S}^{n-1} \times$ 
$\mathbf{S}^{m-1}, v>-1$ and

$$
M_{q}^{(0, v)}\left(\left\{C_{\mu}\right\}\right)=\sum_{\mu=1}^{\infty}\left|C_{\mu}\right|\left\{1+\log ^{(v+1)}\left(\left|I_{\mu}\right|^{-1}\right)\right\} .
$$

Now, we need to introduce some notations.

Definition 2.3. For each $\mu \in \mathbf{N} \cup 0\}$ and an interval $I_{\mu}$ on $\mathbf{S}^{n-1} \times \mathbf{S}^{m-1}$ with $\left|I_{\mu}\right|<e^{-1}$, we set $\theta_{\mu}=\log \left|I_{\mu}\right|^{-1}, \omega_{\mu}=2^{\theta_{\mu}}$ and $J_{k, \mu}=\left[\omega_{\mu}^{k}, \omega_{\mu}^{k+1}\right)$. For suitable mappings $\Phi: \mathbb{R}^{n} \backslash\{0\} \longrightarrow \mathbb{R}^{N}$ and $\Psi: \mathbb{R}^{m} \backslash\{0\} \longrightarrow \mathbb{R}^{M}$ and a suitable function $\tilde{b}_{\mu} \in L^{1}\left(\mathbf{S}^{n-1} \times \mathbf{S}^{m-1}\right)$, we define the sequence of measures $\left\{\sigma_{k, j, \Phi, \Psi, \mu}: k, j \in \mathbf{Z}\right\}$ and its corresponding maximal operator $\sigma_{\Phi, \Psi, \mu}^{*}$ by

$$
\begin{aligned}
\int_{\mathbf{R}^{N} \times \mathbf{R}^{M}} f d \sigma_{k, j, \Phi, \Psi, \mu} & =\int_{J_{k, \mu} \times J_{j, \mu}} f(\Phi(u), \Psi(v)) K_{\tilde{b}_{\mu}}(u, v) d v d u, \\
\sigma_{\Phi, \Psi, \mu}^{*}(f) & =\sup _{k, j \in \mathbf{Z}}|| \sigma_{k, j, \Phi, \Psi, \mu}|* f|,
\end{aligned}
$$

where $\left|\sigma_{k, j, \Phi, \Psi, \mu}\right|$ is defined in the same way as $\sigma_{k, j, \Phi, \Psi, \mu}$, but with $\tilde{b}_{\mu}$ replaced by $\left|\tilde{b}_{\mu}\right|$.

Our method in proving our main results relies heavily on certain maximal functions and on certain Fourier transform estimates. So we need to recall some lemmas. We start with the following lemma due to Ricci and Stein.

Lemma 2.4.([14]) Let $\gamma(t)=\left(a_{1} t^{q_{1}}, \ldots, a_{n} t^{q_{n}}\right)$ where $a_{l}, q_{l} \in \mathbf{R}$ for $1 \leq l \leq n$. Let $\mathscr{M}_{\gamma}$ be the maximal operator defined on $\mathbf{R}^{n}$ by

$$
\mathscr{M}_{\gamma} f(x)=\sup _{R>0} \frac{1}{R}\left|\int_{0}^{R} f(x-\gamma(t)) d t\right|
$$

for $x \in \mathbf{R}^{n}$. Then, for $1<p \leq \infty$, there exists a constant $C_{p}>0$ such that

$$
\left\|\mathscr{M}_{\gamma} f\right\|_{p} \leq C_{p}\|f\|_{p}
$$

for all $f$ in $L^{p}\left(\mathbf{R}^{n}\right)$. The constant $C_{p}$ is independent of $a_{l}$ for all $1 \leq l \leq n$.

The following result follows immediately from the Lemma 2.4.

Lemma 2.5. Let $\gamma(t)=\left(a_{1} t^{q_{1}}, \ldots, a_{n} t^{q_{n}}\right), \vartheta(s)=\left(b_{1} s^{r_{1}}, \ldots, b_{m} s^{r_{m}}\right)$ where $a_{l}, q_{l}, b_{s}$ and $r_{s} \in \mathbf{R}$ for $1 \leq l \leq n$ and $1 \leq s \leq m$. Let $\mathscr{M}_{\gamma, \vartheta}$ be the maximal operator defined on $\mathbf{R}^{n} \times \mathbf{R}^{m}$ by

$$
\mathscr{M}_{\gamma, \vartheta} f(x, y)=\sup _{R_{1}, R_{2}>0} \frac{1}{R_{1} R_{2}}\left|\int_{0}^{R_{1}} \int_{0}^{R_{2}} f(x-\gamma(t), y-\vartheta(r)) d t d r\right|
$$

for $(x, y) \in \mathbf{R}^{n} \times \mathbf{R}^{m}$. Then, for $1<p \leq \infty$, there exists a constant $C_{p}>0$ such that

$$
\left\|\mathscr{M}_{\gamma, \vartheta} f\right\|_{p} \leq C_{p}\|f\|_{p}
$$


for all $f$ in $L^{p}\left(\mathbf{R}^{n} \times \mathbf{R}^{m}\right)$. The constant $C_{p}$ is independent of $a_{l}$ and $b_{s}$ for all $1 \leq l \leq n$ and $1 \leq s \leq m$.

Let $\Gamma: \mathbf{R}^{+} \rightarrow \mathbf{R}$ be a generalized polynomial defined by

$$
\Gamma(t)=t^{a_{1}}+\mu_{2} t^{a_{2}}+\cdots+\mu_{n} t^{a_{n}},
$$

where $\mu_{2}, \ldots, \mu_{n}$ are real parameters and $a_{1}, \ldots, a_{n}$ are real numbers.

Lemma 2.6.([13]) Let $\psi \in C^{1}[0,1]$ and $\Gamma$ be given by (2.2) with $a_{1}, \ldots, a_{n}$ are distinct positive (not necessarily integers) exponents. If

$$
I(\lambda)=\int_{\alpha}^{\beta} e^{i \lambda \Gamma(t)} \psi(t) d t
$$

then

$$
|I(\lambda)| \leq C|\lambda|^{-\varepsilon}\left\{\sup _{\alpha \leq t \leq \beta}|\psi(t)| d t+\int_{\alpha}^{\beta}\left|\psi^{\prime}(t)\right| d t\right\},
$$

where $\lambda \in \mathbf{R} \backslash\{0\}, \varepsilon=\min \left\{1 / a_{1}, 1 / n\right\}$ and $C$ does not depend on $\mu_{2}, \ldots, \mu_{n}$ as long as $0 \leq \alpha<\beta \leq$ 1.

By Lemma 2.6 and the change of variable $t \rightarrow 1 / t$ we immediately get the following:

Lemma 2.7. Let $\psi \in C^{1}[1,2]$ and $\Gamma$ be given by (2.2) with $a_{1}, \ldots, a_{n}$ are distinct negative (not necessarily integers) exponents. If

$$
I(\lambda)=\int_{\alpha}^{\beta} e^{i \lambda \Gamma(t)} \psi(t) d t, 1 \leq \alpha<\beta \leq 2,
$$

then

$$
|I(\lambda)| \leq C|\lambda|^{-\delta}\left\{\sup _{\alpha \leq t \leq \beta}|\varphi(t)| d t+\int_{\alpha}^{\beta}\left|\varphi^{\prime}(t)\right| d t\right\},
$$

where $\lambda \in \mathbf{R} \backslash\{0\}, \delta=\min \left\{-1 / a_{1}, 1 / n\right\}, \varphi(t)=t^{-2} \psi(1 / t)$ and $C$ does not depend on $\mu_{2}, \ldots, \mu_{n}$.

By an argument which is similar to the proof of lemma 3 in [13] we get the following:

Lemma 2.8. Let $\psi \in C^{1}([1 / 2,1])$ and

$$
\Lambda(t)=t^{a_{1}}+\mu_{2} t^{a_{2}}+\cdots+\mu_{k} t^{a_{k}}+\mu_{k+1} t^{-a_{k+1}}+\cdots+\mu_{n} t^{-a_{n}},
$$

where $\mu_{2}, \ldots, \mu_{n}$ are real parameters and $a_{1}, \ldots, a_{n}$ are distinct positive exponents. Let

$$
I(\lambda)=\int_{\alpha}^{\beta} e^{i \lambda \Lambda(t)} \psi(t) d t
$$

$\lambda \in \mathbf{R} \backslash\{0\}$ and $1 / 2<\alpha<\beta \leq 1$. Then

$$
|I(\lambda)| \leq C|\lambda|^{-\varepsilon}\left\{\sup _{\alpha \leq t \leq \beta}|\psi(t)|+\int_{\alpha}^{\beta}\left|\psi^{\prime}(t)\right| d t\right\},
$$


with $\varepsilon=\min \left\{1 / a_{1}, 1 / n\right\}$, where $C$ does not depend on $\mu_{2}, \ldots, \mu_{n}$ and $\lambda$.

We shall need the following lemma from [4]:

Lemma 2.9. For $j \in\{1,2\}$, let $U_{j}$ be a domain in $\mathbf{R}^{n_{j}}$ and $K_{j}$ a compact subset of $U_{j}$. Let $g(\cdot, \cdot)$ be a real-analytic function on $U_{1} \times U_{2}$ such that $g(\cdot, y)$ is a nonzero function for every $y \in U_{2}$. Then there exist a positive constant $\delta=\delta\left(h, K_{1}, K_{2}\right)$ such that

$$
\sup _{y \in K_{2}} \int_{K_{1}}|g(x, y)|^{-\delta} d x<\infty
$$

By tracking the constants in the proof of Lemma 1 in [5] we have the following:

Lemma 2.10. Let $A>0$ and let $\left\{v_{k, j}\right\}$ be a sequence of Borel measures on $\mathbf{R}^{n} \times \mathbf{R}^{m}$. Suppose that

$$
\left\|\sup _{k, j \in \mathbf{Z}}|| v_{k, j}|* f|\right\|_{q_{0}} \leq A\|f\|_{q_{0}}
$$

for some $q_{0}>1$ and for every $f$ in $L^{q_{0}}\left(\mathbf{R}^{n} \times \mathbf{R}^{m}\right)$. Then the inequality

$$
\left\|\left(\sum_{k, j \in \mathbf{Z}}\left|v_{k, j} * g_{k, j}\right|^{2}\right)^{1 / 2}\right\|_{p_{0}} \leq\left(A \sup _{k, j \in \mathbf{Z}}\left\|v_{k, j}\right\|\right)^{1 / 2}\left\|\left(\sum_{k, j \in \mathbf{Z}}\left|g_{k, j}\right|^{2}\right)^{1 / 2}\right\|_{p_{0}}
$$

holds for $\left|1 / p_{0}-1 / 2\right|=1 /\left(2 q_{0}\right)$ and for arbitrary functions $\left\{g_{k, j}\right\}$ on $\mathbf{R}^{n} \times \mathbf{R}^{m}$.

The proof of Theorem 1.1 relies heavily on the following lemma which is a generalization of a result of J. Duoandikoetxea [5]. A proof of this lemma can obtained directly from Lemma 2.10 and Theorem 16 in [3].

Lemma 2.11. Let $M, N \in \mathbf{N}$ and let $\left\{\sigma_{k, j}^{(l, s)}: k, j \in \mathbf{Z}, 0 \leq l \leq N, 0 \leq s \leq M\right\}$ be a family of Borel measures on $\mathbf{R}^{n} \times \mathbf{R}^{m}$ with $\sigma_{k, j}^{(l, M)}=0$ and $\sigma_{k, j}^{(N, s)}=0$ for $k, j \in \mathbf{Z}$. Let $\left\{a_{l}, b_{s}: 0 \leq l \leq N-1,0 \leq s \leq\right.$ $M-1\} \subset[2, \infty),\{b(l), d(s): 0 \leq l \leq N-1,0 \leq s \leq M-1\} \subset \mathbf{N},\left\{\alpha_{l}, \beta_{s}: 0 \leq l \leq N-1,0 \leq s \leq M-1\right\} \subseteq$ $\mathbf{R}^{+}$, and let $L^{(l)} \in L\left(\mathbf{R}^{n}, \mathbf{R}^{b(l)}\right)$ and $Q^{(s)} \in L\left(\mathbf{R}^{m}, \mathbf{R}^{d(s)}\right)$ be for $0 \leq l \leq N-1$ and $0 \leq s \leq M-1$, where $L\left(\mathbf{R}^{n}, \mathbf{R}^{N}\right)$ denotes the space of linear transformations from $\mathbf{R}^{n}$ into $\mathbf{R}^{N}$. Suppose that for some $C>0$ and $B>1$, the following hold for $k, j \in \mathbf{Z}, 0 \leq l \leq N-1,0 \leq s \leq M-1$ and $(\xi, \eta) \in \mathbf{R}^{n} \times \mathbf{R}^{m}:$

(i) $\left\|\sigma_{k, j}^{(l, s)}\right\| \leq C B^{2}$;

(ii) $\left|\hat{\sigma}_{k, j}^{(l, s)}(\xi, \eta)\right| \leq C B^{2}\left|a_{l}^{k B} L^{(l)}(\xi)\right|^{-\frac{\alpha_{l}}{B}}\left|b_{s}^{j B} Q^{(s)}(\eta)\right|^{-\frac{\beta_{s}}{B}}$;

(iii) $\left|\hat{\sigma}_{k, j}^{(l, s)}(\xi, \eta)-\hat{\sigma}_{k, j}^{(l+1, s)}(\xi, \eta)\right| \leq C B^{2}\left|a_{l}^{k B} L^{(l)}(\xi)\right|^{\frac{\alpha_{l}}{B}}\left|b_{s}^{j B} Q^{(s)}(\eta)\right|^{-\frac{\beta_{s}}{B}}$;

(iv) $\left|\hat{\sigma}_{k, j}^{(l, s)}(\xi, \eta)-\hat{\sigma}_{k, j}^{(l, s+1)}(\xi, \eta)\right| \leq C B^{2}\left|a_{l}^{k B} L^{(l)}(\xi)\right|^{-\frac{\alpha_{l}}{B}}\left|b_{s}^{j B} Q^{(s)}(\eta)\right|^{\frac{\beta_{s}}{B}}$; 
(v) $\left|\hat{\sigma}_{k, j}^{(l, s)}(\xi, \eta)-\hat{\sigma}_{k, j}^{(l+1, s)}(\xi, \eta)-\hat{\sigma}_{k, j}^{(l, s+1)}(\xi, \eta)+\hat{\sigma}_{k, j}^{(l+1, s+1)}(\xi, \eta)\right|$

$$
\leq C B^{2}\left|a_{l}^{k B} L^{(l)}(\xi)\right|^{\frac{\alpha_{l}}{B}}\left|b_{s}^{j B} Q^{(s)}(\eta)\right|^{\frac{\beta_{s}}{B}}
$$

(vi) $\left|\hat{\sigma}_{k, j}^{(l, s+1)}(\xi, \eta)-\hat{\sigma}_{k, j}^{(l+1, s+1)}(\xi, \eta)\right| \leq C B^{2}\left|a_{l}^{k B} L^{(l)}(\xi)\right|^{\frac{\alpha_{l}}{B}}$;

(vii) $\left|\hat{\sigma}_{k, j}^{(l+1, s)}(\xi, \eta)-\hat{\sigma}_{k, j}^{(l+1, s+1)}(\xi, \eta)\right| \leq C B^{2}\left|b_{s}^{j B} Q^{(s)}(\eta)\right|^{\frac{\beta_{s}}{B}}$;

(viii) $\left\|\sup _{k, j \in \mathbf{Z}}|| \sigma_{k, j}^{(l, s)}|* f|\right\|_{p} \leq C B^{2}\|f\|_{p}$ for $1<p<\infty$ and for every $f$ in $L^{p}\left(\mathbf{R}^{n} \times \mathbf{R}^{m}\right)$. Then for every $1<p<\infty$, there exists a $C_{p}>0$ independent of $\left\{L^{(l)}, Q^{(s)}: 0 \leq l \leq N-1,0 \leq s \leq\right.$ $M-1\}$ such that

$$
\left\|\sum_{k, j \in \mathbf{Z}} \sigma_{k, j}^{(0,0)} * f\right\|_{p} \leq C_{p} B^{2}\|f\|_{p}
$$

holds for all $f$ in $L^{p}\left(\mathbf{R}^{n} \times \mathbf{R}^{m}\right)$.

Lemma 2.12. Let $N, M \in \mathbf{N}$, let $\tilde{b}_{\mu}$ be a function on $\mathbf{S}^{n-1} \times \mathbf{S}^{m-1}$ satisfying (i) $\left\|\tilde{b}_{\mu}\right\|_{q} \leq$ $\left|I_{\mu}\right|^{-1 / q^{\prime}}$ for some $q>1$ and an interval $I_{\mu}$ on $\mathbf{S}^{n-1} \times \mathbf{S}^{m-1}$ with $\left|I_{\mu}\right|<e^{-1}$ and (ii) $\left\|\tilde{b}_{\mu}\right\|_{1} \leq 1$. Let $\Phi: \mathbf{R}^{n} \longrightarrow \mathbf{R}^{N}$ and $\Psi: \mathbf{R}^{m} \longrightarrow \mathbf{R}^{M}$ be homogeneous mappings of degrees $d=\left(d_{1}, \ldots, d_{N}\right)$ and $h=\left(h_{1}, \ldots, h_{M}\right)$, respectively with $d_{l}, h_{s}>0$ for $1 \leq l \leq N$ and $1 \leq s \leq M$. Assume that $\Phi \mid \mathbf{S}^{n-1}$ and $\Psi \mid \mathbf{S}^{m-1}$ are real-analytic and that there are $z_{1}, \tilde{z}_{1}, w_{1}$ and $\tilde{w}_{1} \in \mathbf{N}$ such that $z_{1} \leq$ $\tilde{z}_{1} \leq N,\left\{l: 1 \leq l \leq N\right.$ and $\left.d_{l}=d_{1}\right\}=\left\{1, \ldots, \tilde{z}_{1}\right\}, w_{1} \leq \tilde{w}_{1} \leq M,\left\{s: 1 \leq s \leq M\right.$ and $\left.h_{s}=h_{1}\right\}=$ $\left\{1, \ldots, \tilde{w}_{1}\right\},\left\{\Phi_{1}, \ldots, \Phi_{z_{1}}\right\}$ forms a basis for $\operatorname{span}\left\{\Phi_{1}, \ldots, \Phi_{\tilde{z}_{1}}\right\}$ and $\left\{\Psi_{1}, \ldots, \Psi_{w_{1}}\right\}$ forms a basis for $\operatorname{span}\left\{\Psi_{1}, \ldots, \Psi_{\tilde{w}_{1}}\right\}$. Then there exist $L \in L\left(\mathbf{R}^{\tilde{z}_{1}}, \mathbf{R}^{z_{1}}\right), Q \in L\left(\mathbf{R}^{\tilde{w}_{1}}, \mathbf{R}^{w_{1}}\right)$ and positive constants $\alpha$, $\beta$ and $C$ such that

$$
\left|\hat{\sigma}_{k, j, \Phi, \Psi, \mu}(\xi, \eta)\right| \leq C \theta_{\mu}^{2}\left|\omega_{\mu}^{k d_{1}} L\left(\Pi_{\tilde{z}_{1}} \xi\right)\right|^{-\frac{\alpha}{\theta_{\mu}}}\left|\omega_{\mu}^{j h_{1}} Q\left(\Pi_{\tilde{w}_{1}} \eta\right)\right|^{-\frac{\beta}{\theta_{\mu}}}
$$

for all $(\xi, \eta) \in \mathbf{R}^{N} \times \mathbf{R}^{M}$, where $\Pi_{\tilde{z}_{1}} \xi=\left(\xi_{1}, \ldots, \xi_{\tilde{z}_{1}}\right)$ and $\Pi_{\tilde{w}_{1}} \eta=\left(\eta_{1}, \ldots, \eta_{\tilde{w}_{1}}\right)$.

Proof. Let $\xi=\left(\xi_{1}, \ldots, \xi_{N}\right)$ and $\eta=\left(\eta_{1}, \ldots, \eta_{M}\right)$ be arbitrary but fixed. By assumptions, there exist two linear transformations $L=\left(L_{1}, \ldots, L_{z_{1}}\right) \in L\left(\mathbf{R}^{\tilde{z}_{1}}, \mathbf{R}^{z_{1}}\right)$ and $Q=\left(Q_{1}, \ldots, Q_{w_{1}}\right) \in$ $L\left(\mathbf{R}^{\tilde{w}_{1}}, \mathbf{R}^{w_{1}}\right)$ such that

$$
\sum_{l=1}^{\tilde{z}_{1}} \xi_{l} \Phi_{l}(x)=\sum_{l=1}^{z_{1}} L_{l}\left(\Pi_{\tilde{z}_{1}} \xi\right) \Phi_{l}(x) \text { and } \sum_{s=1}^{\tilde{w}_{1}} \eta_{s} \Psi_{s}(y)=\sum_{s=1}^{w_{1}} Q_{s}\left(\Pi_{\tilde{w}_{1}} \eta\right) \Psi_{s}(y)
$$

Thus we have

$$
\left|\hat{\sigma}_{k, j, \Phi, \Psi, \mu}(\xi, \eta)\right| \leq C \theta_{\mu} \int_{\mathbf{S}^{n-1} \times \mathbf{S}^{m-1}}\left|\tilde{b}_{\mu}(x, y)\right|\left|\int_{1 / \omega_{\mu}}^{1} e^{-i H_{\xi, k}(t, x)} \frac{d t}{t}\right| d \sigma(x) d \sigma(y),
$$

where

$$
H_{\xi, k}(t, x)=\left(\sum_{l=1}^{\tilde{z}_{1}} \xi_{l} \Phi_{l}(x)\right) t^{d_{1}} \omega_{\mu}^{(k+1) d_{1}}+\sum_{s=\tilde{z}_{1}+1}^{N} \xi_{s} \Phi_{s}(x) t^{d_{s}} \omega_{\mu}^{(k+1) d_{s}}
$$


Let $g: \mathbf{S}^{n-1} \times \mathbf{S}^{z_{1}-1} \rightarrow \mathbf{R}$ be given by

$$
g(x, u)=\sum_{l=1}^{z_{1}} u_{l} \Phi_{l}(x),
$$

where $x \in \mathbf{S}^{n-1}$ and $u=\left(u_{1}, \ldots, u_{z_{1}}\right) \in \mathbf{S}^{z_{1}-1}$. Since $\left\{\Phi_{1}, \ldots, \Phi_{z_{1}}\right\}$ is linearly independent, $g(\cdot, u)$ is a nonzero function for every $u \in \mathbf{S}^{z_{1}-1}$. By Lemma 2.9, there exists a $\delta_{1}>0$ such that

$$
\sup _{u \in \mathbf{S}^{z_{1}-1}} \int_{\mathbf{S}^{n-1}}|g(x, u)|^{-\delta_{1}} d \sigma(x)<\infty .
$$

By letting $\varepsilon=\min \left\{1 / d_{1}, 1 / N, \delta_{1} / q^{\prime}\right\},(2.8)$, (i), using Lemma 2.6 and Hölder's inequality we get

$$
\begin{aligned}
\left|\hat{\sigma}_{k, j, \Phi, \Psi, \mu}(\xi, \eta)\right| & \leq C \theta_{\mu} \int_{\mathbf{S}^{n-1} \times \mathbf{S}^{m-1}}\left|\tilde{b}_{\mu}(x, y)\right|\left|\sum_{l=1}^{z_{1}} L_{l}\left(\Pi_{\tilde{z}_{1}} \xi\right) \Phi_{l}(x)\right|^{-\varepsilon} d \sigma(x) d \sigma(y) \\
& \leq C \theta_{\mu} \omega_{\mu}^{-\varepsilon d_{1}} \|\left.\tilde{b}_{\mu}\right|_{L^{q}\left(\mathbf{S}^{n-1} \times \mathbf{S}^{m-1}\right)}\left|\omega_{\mu}^{k d_{1}} L\left(\Pi_{\tilde{z}_{1}} \xi\right)\right|^{-\epsilon} \\
& \leq C \theta_{\mu} \omega_{\mu}^{-\varepsilon d_{1}}\left|I_{\mu}\right|^{-1 / q^{\prime}}\left|\omega_{\mu}^{k d_{1}} L\left(\Pi_{\tilde{z}_{1}} \xi\right)\right|^{-\epsilon} .
\end{aligned}
$$

By combining the last estimate with the trivial estimate $\left|\hat{\sigma}_{k, j, \Phi, \Psi, \mu}(\xi, \eta)\right| \leq C \theta_{\mu}^{2}$ we get

$$
\left|\hat{\sigma}_{k, j, \Phi, \Psi, \mu}(\xi, \eta)\right| \leq C \theta_{\mu}^{2}\left|\omega_{\mu}^{k d_{1}} L\left(\Pi_{\tilde{z}_{1}} \xi\right)\right|^{-\epsilon / \theta_{\mu}}
$$

Similarly, we have

$$
\left|\hat{\sigma}_{k, j, \Phi, \Psi, \mu}(\xi, \eta)\right| \leq C \theta_{\mu}^{2}\left|\omega_{\mu}^{j h_{1}} Q\left(\Pi_{\tilde{w}_{1}} \eta\right)\right|^{-\beta / \theta_{\mu}} .
$$

Combining the last two estimates yields the desired estimate. The proof is complete. 


\section{Proof of Theorem 1.1}

Assume that $\Omega \in B_{q}^{(0,1)}\left(\mathbf{S}^{n-1} \times \mathbf{S}^{m-1}\right)$ for some $q>1$ and satisfies (1.2). Thus $\Omega$ can be written as $\Omega=\sum_{\mu=1}^{\infty} C_{\mu} b_{\mu}$, where $C_{\mu} \in \mathbf{C}, b_{\mu}$ is a $q$-block supported on an interval $I_{\mu}$ on $\mathbf{S}^{n-1} \times$ $\mathbf{S}^{m-1}$ and $M_{q}^{(0,1)}\left(\left\{C_{\mu}\right\}\right)<\infty$. To each block function $b_{\mu}(\cdot, \cdot)$, let $\tilde{b}_{\mu}(\cdot, \cdot)$ be a function defined by

$$
\begin{aligned}
\tilde{b}_{\mu}(x, y)= & b_{\mu}(x, y)-\int_{\mathbf{S}^{n-1}} b_{\mu}(u, y) d \sigma(u) \\
& -\int_{\mathbf{S}^{m-1}} b_{\mu}(x, v) d \sigma(v)+\int_{\mathbf{S}^{n-1} \times \mathbf{S}^{m-1}} b_{\mu}(u, v) d \sigma(u) d \sigma(v) .
\end{aligned}
$$

Let $\mathbf{D}=\left\{\mu \in \mathbf{N}:\left|I_{\mu}\right|<e^{-1}\right\}$. Let $\tilde{b}_{0}=\Omega-\sum_{\mu \in \mathbf{D}} C_{\mu} \tilde{b}_{\mu}$. Then it is easy to verify that, for all $\mu \in \mathbf{D} \cup\{0\}$, $\tilde{b}_{\mu}$ satisfies the following:

$$
\begin{aligned}
\int_{\mathbf{S}^{n-1}} \tilde{b}_{\mu}(u, \cdot) d \sigma(u) & =\int_{\mathbf{S}^{m-1}} \tilde{b}_{\mu}(\cdot, v) d \sigma(v)=0 \\
\left\|\tilde{b}_{\mu}\right\|_{q} & \leq C\left|I_{\mu}\right|^{-1 / q^{\prime}} \\
\left\|\tilde{b}_{\mu}\right\|_{1} & \leq C
\end{aligned}
$$

where $\left|I_{0}\right|=e^{-2}$ and $C$ is a positive constant independent of $\mu$. Using the assumption that $\Omega$ satisfies the vanishing conditions (1.2), and the definition of $\tilde{b}_{\mu}$, we deduce that $\Omega$ can be written as

$$
\Omega=\sum_{\mu \in \mathbf{D} \cup\{0\}} C_{\mu} \tilde{b}_{\mu}
$$

which in turn implies

$$
\begin{aligned}
& T_{\Phi, \Psi, \Omega}(f)=\sum_{\mu \in \mathbf{D} \cup\{0\}} C_{\mu} T_{\Phi, \Psi, \tilde{b}_{\mu}}(f), \\
& T_{\Phi, \Psi, \Omega}^{*}(f) \leq \sum_{\mu \in \mathbf{D} \cup\{0\}}\left|C_{\mu}\right| T_{\Phi, \Psi, \tilde{b}_{\mu}}^{*}(f) .
\end{aligned}
$$

Therefore, to prove (1.5)-(1.6), it suffices to prove the following inequalities:

$$
\begin{gathered}
\left\|T_{\Phi, \Psi, \tilde{b}_{\mu}}(f)\right\|_{p} \leq C_{p} \theta_{\mu}^{2}\|f\|_{p}, \\
\left\|T_{\Phi, \Psi, \tilde{b}_{\mu}}^{*}(f)\right\|_{p} \leq C_{p} \theta_{\mu}^{2}\|f\|_{p}
\end{gathered}
$$

for $1<p<\infty$ and $\mu \in \mathbf{D} \cup\{0\}$. Let us start with proving (3.4). By assumptions on $\Phi$ and $\Psi$, we have $\Phi=\left(\Phi_{1}, \ldots, \Phi_{N}\right): \mathbf{R}^{n} \rightarrow \mathbf{R}^{N}$ and $\Psi=\left(\Psi_{1}, \ldots, \Psi_{M}\right): \mathbf{R}^{m} \rightarrow \mathbf{R}^{M}$ are homogeneous mappings of degrees $d=\left(d_{1}, \ldots, d_{N}\right)$ and $h=\left(h_{1}, \ldots, h_{M}\right)$, respectively such that $\Phi \mid \mathbf{S}^{n-1}$ and $\Psi \mid \mathbf{S}^{m-1}$ are real-analytic and $d_{l}, h_{s} \neq 0$ for $1 \leq l \leq N$ and $1 \leq s \leq M$. In view of Lemmas 2.6-2.8, we 
shall only prove (3.4) only for the case $d_{1}, \ldots, d_{N}, h_{1}, \ldots, h_{M}>0$ because the argument for the other cases will be similar and requires only minor modifications. Now, we use an argument employed in [4] and [1]. By a simple reordering of the mappings $\Phi_{1}, \ldots, \Phi_{N}, \Psi_{1}, \ldots, \Psi_{M}$ we may assume that there are $z_{1}, \tilde{z}_{1}, w_{1}$ and $\tilde{w}_{1} \in \mathbf{N}$ such that $z_{1} \leq \tilde{z}_{1} \leq N,\{l: 1 \leq l \leq N$ and $\left.d_{l}=d_{1}\right\}=\left\{1, \ldots, \tilde{z}_{1}\right\}, w_{1} \leq \tilde{w}_{1} \leq M,\left\{s: 1 \leq s \leq M\right.$ and $\left.h_{s}=h_{1}\right\}=\left\{1, \ldots, \tilde{w}_{1}\right\},\left\{\Phi_{1}, \ldots, \Phi_{z_{1}}\right\}$ forms a basis for $\operatorname{span}\left\{\Phi_{1}, \ldots, \Phi_{\tilde{z}_{1}}\right\}$ and $\left\{\Psi_{1}, \ldots, \Psi_{w_{1}}\right\}$ forms a basis for $\operatorname{span}\left\{\Psi_{1}, \ldots, \Psi_{\tilde{w}_{1}}\right\}$.

Let $\Gamma_{0}=\Phi, \Gamma_{1}=\left(0, \ldots, 0, \Phi_{\tilde{z}_{1}+1}, \ldots, \Phi_{N}\right), L^{(0)}(\xi)=L\left(\Pi_{\tilde{z}_{1}} \xi\right)$ for $\xi \in \mathbf{R}^{N}, \Upsilon_{0}=\Psi, \Upsilon_{1}=\left(0, \ldots, 0, \Psi_{\tilde{w}_{1}+1}, \ldots, \Psi_{M}\right), Q^{(0)}(\eta)=$ $Q\left(\Pi_{\tilde{w}_{1}} \eta\right)$ for $\eta \in \mathbb{R}^{M}$, and $\sigma_{k, j, \mu}^{(l, s)}=\sigma_{k, j, \Gamma_{l}, Y_{s}, \mu}$ for $l, s \in\{0,1\}$. By invoking (3.1)-(3.3) and Lemma 2.12 we get

$$
\begin{aligned}
& \left|\sigma_{k, j, \mu}^{(l, s)}(\xi, \eta)\right| \leq C \theta_{\mu}^{2} \text { for } l, s \in\{0,1\} ; \\
& \left|\hat{\sigma}_{k, j, \mu}^{(0,0)}(\xi, \eta)\right| \leq C \theta_{\mu}^{2}\left|\omega_{\mu}^{k d_{1}} L^{(0)}(\xi)\right|^{-\frac{\alpha_{0}}{\theta_{\mu}}}\left|\omega_{\mu}^{j h_{1}} Q^{(0)}(\eta)\right|^{-\frac{\beta_{0}}{\theta_{\mu}}} .
\end{aligned}
$$

Also,

$$
\begin{gathered}
\left|\hat{\sigma}_{k, j, \mu}^{(0,0)}(\xi, \eta)-\hat{\sigma}_{k, j, \mu}^{(0,1)}(\xi, \eta)\right| \leq \int_{1 / \omega_{\mu}}^{1} \int_{\mathbf{S}^{n-1} \times \mathbf{S}^{m-1}}\left|\tilde{b}_{\mu}(x, y)\right| \times \\
\left|\int_{1 / \omega_{\mu}}^{1} e^{-i H_{\xi, k}(t, x)} \frac{d t}{t}\right|\left|e^{-i \eta \cdot \Upsilon_{0}\left(\omega_{\mu}^{j+1} s y\right)}-e^{-i \eta \cdot \Upsilon_{1}\left(\omega_{\mu}^{j+1} s y\right)}\right| d \sigma(x) d \sigma(y) \frac{d s}{s},
\end{gathered}
$$

where $H_{\xi, k}(t, x)$ is given by (2.7). By a similar argument as that employed in the proof of (2.9) we get

$$
\left|\hat{\sigma}_{k, j, \mu}^{(0,0)}(\xi, \eta)-\hat{\sigma}_{k, j, \mu}^{(0,1)}(\xi, \eta)\right| \leq C \theta_{\mu} \omega_{\mu}^{-\varepsilon d_{1}}\left|I_{\mu}\right|^{-1 / q^{\prime}}\left|\omega_{\mu}^{(j+1) h_{1}} Q^{(0)}(\eta)\right|\left|\omega_{\mu}^{k d_{1}} L\left(\Pi_{\tilde{z}_{1}} \xi\right)\right|^{-\varepsilon}
$$

which when combined with the trivial estimate $\left|\hat{\sigma}_{k, j, \mu}^{(0,0)}(\xi, \eta)-\hat{\sigma}_{k, j, \mu}^{(0,1)}(\xi, \eta)\right| \leq C \theta_{\mu}^{2}$ yields

$$
\left|\hat{\sigma}_{k, j, \mu}^{(0,0)}(\xi, \eta)-\hat{\sigma}_{k, j, \mu}^{(0,1)}(\xi, \eta)\right| \leq C \theta_{\mu}^{2}\left|\omega_{\mu}^{k d_{1}} L^{(0)}(\xi)\right|^{-\frac{\alpha_{0}}{\theta_{\mu}}}\left|\omega_{\mu}^{j h_{1}} Q^{(0)}(\eta)\right|^{\frac{\beta_{0}}{\theta_{\mu}}} .
$$

Similarly, we get

$$
\begin{gathered}
\left|\hat{\sigma}_{k, j, \mu}^{(0,0)}(\xi, \eta)-\hat{\sigma}_{k, j, \mu}^{(1,0)}(\xi, \eta)\right| \leq C \theta_{\mu}^{2}\left|\omega_{\mu}^{k d_{1}} L^{(0)}(\xi)\right|^{\frac{\alpha_{0}}{\theta_{\mu}}}\left|\omega_{\mu}^{j h_{1}} Q^{(0)}(\eta)\right|^{-\frac{\beta_{0}}{\theta_{\mu}}} ; \\
\left|\hat{\sigma}_{k, j, \mu}^{(0,1)}(\xi, \eta)-\hat{\sigma}_{k, j, \mu}^{(1,1)}(\xi, \eta)\right| \leq C \theta_{\mu}^{2}\left|\omega_{\mu}^{k d_{1}} L^{(0)}(\xi)\right|^{\frac{\alpha_{0}}{\theta_{\mu}}} ; \\
\left|\hat{\sigma}_{k, j, \mu}^{(1,0)}(\xi, \eta)-\hat{\sigma}_{k, j, \mu}^{(1,1)}(\xi, \eta)\right| \leq C \theta_{\mu}^{2}\left|\omega_{\mu}^{j h_{1}} Q^{(0)}(\eta)\right|^{\frac{\beta_{0}}{\theta_{\mu}}} ; \\
\left|\hat{\sigma}_{k, j, \mu}^{(0,0)}(\xi, \eta)-\hat{\sigma}_{k, j, \mu}^{(0,1)}(\xi, \eta)-\hat{\sigma}_{k, j, \mu}^{(1,0)}(\xi, \eta)+\hat{\sigma}_{k, j, \mu}^{(1,1)}(\xi, \eta)\right| \\
\leq C \theta_{\mu}^{2}\left|\omega_{\mu}^{k d_{1}} L^{(0)}(\xi)\right|^{\frac{\alpha_{0}}{\theta_{\mu}}}\left|\omega_{\mu}^{j h_{1}} Q^{(0)}(\eta)\right|^{\frac{\beta_{0}}{\theta_{\mu}}}
\end{gathered}
$$


Now, by a similar argument as that employed above, we can find additional mappings $\Gamma_{2}, \ldots$, $\Gamma_{K}$ from $\mathbf{R}^{n} \backslash\{0\}$ to $\mathbf{R}^{N}, \Upsilon_{2}, \ldots, \Upsilon_{J}$ from $\mathbf{R}^{m} \backslash\{0\}$ to $\mathbf{R}^{M},\left\{\alpha_{l}, \beta_{s}: 1 \leq l \leq K-1,1 \leq s \leq J-1\right\} \subset$ $(0, \infty)$, appropriate linear transformations $\left\{L^{(l)}, Q^{(s)}: 1 \leq l \leq K-1,1 \leq s \leq J-1\right\}$, two sets of distinct real numbers $\left\{d_{u_{l}}: 1 \leq l \leq K-1\right\},\left\{h_{\nu_{s}}: 1 \leq s \leq J-1\right\}$ with $\left\{d_{u_{l}}: 1 \leq l \leq K-1\right\}=\left\{d_{l}\right.$ : $2 \leq l \leq N\} \backslash\left\{d_{1}\right\},\left\{h_{\nu s}: 1 \leq s \leq J-1\right\}=\left\{h_{s}: 2 \leq s \leq M\right\} \backslash\left\{h_{1}\right\}$ and a finite family of measures $\left\{\sigma_{k, j, \mu}^{(l, s)}: 2 \leq l \leq K, 2 \leq s \leq J\right\}$ with the following properties:

$$
\begin{aligned}
& \Gamma_{K}=(0, \ldots, 0), \Upsilon_{J}=(0, \ldots, 0) ; \\
& \sigma_{k, j, \mu}^{(l, s)}(\xi, \eta)=\sigma_{k, j, \mu, \Gamma_{l}, \Upsilon_{s}} \text { for } 2 \leq l \leq K \text { and } 2 \leq s \leq J \\
& \sigma_{k, j, \mu}^{(K, s)}=\sigma_{k, j, \mu}^{(l, J)}=0 \text { for } 2 \leq l \leq K \text { and } 2 \leq s \leq J \\
& \left|\sigma_{k, j, \mu}^{(l, s)}(\xi, \eta)\right| \leq C \theta_{\mu}^{2} \\
& \left|\hat{\sigma}_{k, j, \mu}^{(l, s)}(\xi, \eta)\right| \leq C \theta_{\mu}^{2}\left|\omega_{\mu}^{k d_{u_{l}}} L^{(l)}(\xi)\right|^{-\frac{\alpha_{l}}{\theta_{\mu}}}\left|\omega_{\mu}^{j h_{\nu s}} Q^{(s)}(\eta)\right|^{-\frac{\beta_{s}}{\theta_{\mu}}} \\
& \left|\hat{\sigma}_{k, j, \mu}^{(l, s)}(\xi, \eta)-\hat{\sigma}_{k, j, \mu}^{(l+1, s)}(\xi, \eta)\right| \leq C \theta_{\mu}^{2}\left|\omega_{\mu}^{k d_{u_{l}}} L^{(l)}(\xi)\right|^{\frac{\alpha_{l}}{\theta_{\mu}}}\left|\omega_{\mu}^{j h_{\nu s}} Q^{(s)}(\eta)\right|^{-\frac{\beta_{s}}{\theta_{\mu}}} ; \\
& \left|\hat{\sigma}_{k, j, \mu}^{(l, s)}(\xi, \eta)-\hat{\sigma}_{k, j, \mu}^{(l, s+1)}(\xi, \eta)\right| \leq C \theta_{\mu}^{2}\left|\omega_{\mu}^{k d_{u_{l}}} L^{(l)}(\xi)\right|^{-\frac{\alpha_{l}}{\theta_{\mu}}}\left|\omega_{\mu}^{j h_{v s}} Q^{(s)}(\eta)\right|^{\frac{\beta_{s}}{\theta_{\mu}}} ; \\
& \left|\hat{\sigma}_{k, j, \mu}^{(l, s+1)}(\xi, \eta)-\hat{\sigma}_{k, j, \mu}^{(l+1, s+1)}(\xi, \eta)\right| \leq C \theta_{\mu}^{2}\left|\omega_{\mu}^{k d_{u_{l}}} L^{(l)}(\xi)\right|^{\frac{\alpha_{l}}{\theta_{\mu}}} \\
& \left|\hat{\sigma}_{k, j, \mu}^{(l+1, s)}(\xi, \eta)-\hat{\sigma}_{k, j, \mu}^{(l+1, s+1)}(\xi, \eta)\right| \leq C \theta_{\mu}^{2}\left|\omega_{\mu}^{j h_{v s}} Q^{(s)}(\eta)\right|^{\frac{\beta_{s}}{\theta_{\mu}}} ; \\
& \left|\hat{\sigma}_{k, j, \mu}^{(l, s)}(\xi, \eta)-\hat{\sigma}_{k, j, \mu}^{(l, s+1)}-\hat{\sigma}_{k, j, \mu}^{(l+1, s)}+\hat{\sigma}_{k, j, \mu}^{(l+1, s+1)}(\xi, \eta)\right| \\
& \leq C \theta_{\mu}^{2}\left|\omega_{\mu}^{k d_{u_{l}}} L^{(l)}(\xi)\right|^{\frac{\alpha_{l}}{\theta_{\mu}}}\left|\omega_{\mu}^{j h_{\nu s}} Q^{(s)}(\eta)\right|^{\frac{\beta_{s}}{\theta_{\mu}}}
\end{aligned}
$$

for $1 \leq l \leq K-1$ and $1 \leq s \leq J-1$. By (3.3) and applying Lemma 2.5 we obtain

$$
\left\|\sup _{k, j \in Z}\right\| \sigma_{k, j, \mu}^{(l, s)} * f\|\|_{p} \leq C_{p} \theta_{\mu}^{2}\|f\|_{p}
$$

for $1<p<\infty, 0 \leq l \leq K-1$ and $0 \leq s \leq J-1$. By (3.6)-(3.20), Lemma 2.11 we have

$$
\left\|T_{\Phi, \Psi, \tilde{b}_{\mu}} f\right\|_{p}=\left\|\sum_{k, j \in \mathbf{Z}} \sigma_{k, j, \mu}^{(0,0)} * f\right\|_{p} \leq C_{p} \theta_{\mu}^{2}\|f\|_{p}
$$

for $1<p<\infty$ and $f \in L^{p}\left(\mathbf{R}^{n} \times \mathbf{R}^{m}\right)$ which completes the proof of (3.4).

A proof of (3.5) can be constructed by the above estimates and employing a similar argument employed in the proof of Theorem B in [3]. Details will be omitted. 


\section{References}

[1] H. Al-Qassem and M. Ali, $L^{p}$ boundedness for singular integral operators with $L\left(\log ^{+} L\right)^{2}$ kernels on product spaces, Kyungpook Math. J. 46(2006), 377-387.

[2] H. Al-Qassem, A. Al-Salman and Y. Pan, Singular integrals associated to homogeneous mappings with rough kernels, Hokkaido Math. J. 33(2004), 551-569.

[3] H. AL-Qassem and Y. Pan, $L^{p}$ boundedness for singular integrals with rough kernels on product domains, Hokkaido Math. J. 31(2002), 555-613.

[4] L. Cheng, Singular integrals related to homogeneous mappings, Michigan Math. J. 47(2000), 407416.

[5] J. Duoandikoetxea, Multiple singular integrals and maximal functions along hypersurfaces, Ann. Ins. Fourier (Grenoble) 36 (1986), 185-206.

[6] D. Fan, K. Guo, and Y. Pan, Singular integrals with rough kernels on product spaces, Hokkaido Math. J. 28 (1999), 435-460.

[7] D. Fan, K. Guo, and Y. Pan, $L^{p}$ estimates for singular integrals associated to homogeneous surfaces, J. Reine Angew. Math. 542 (2002), 1-22.

[8] R. Fefferman, Singular integrals on product domains, Bull. Amer. Math. Soc. 4(1981), 195-201.

[9] R. Fefferman and E. M. Stein, Singular integrals on product spaces, Adv. in Math. 45(1982), 117-143.

[10] Y. Jiang and S. Lu, A class of singular integral operators with rough kernels on product domains, Hokkaido Math. J. 24(1995), 1-7.

[11] M. Keitoku and E. Sato, Block spaces on the unit sphere in $\mathbf{R}^{n}$, Proc. Amer. Math. Soc. 119(1993), 453-455.

[12] S. Lu, M. Taibleson and G. Weiss, Spaces Generated by Blocks, Beijing Normal University Press, 1989, Beijing.

[13] F. Ricci and E. M. Stein, Harmonic analysis on nilpotent groups and singular integrals I: Oscillatory integrals, Jour. Func. Anal. 73 (1987), 179-194.

[14] F. Ricci and E. M. Stein, Multiparameter singular integrals and maximal functions, Ann. Inst. Fourier 42 (1992), 637-670.

[15] M. H. Taibleson and G. Weiss, Certain function spaces associated with a.e. convergence of Fourier series, Univ. of Chicago Conf. in honor of Zygmund, Woodsworth, 83.

Department of Mathematics, Yarmouk University, Irbid-Jordan.

E-mail: husseink@yu.edu.jo

E-mail: hamadneh2004@hotmail.com 